\title{
Síndrome ocular isquêmica associada a glaucoma neovas- cular: relato de um caso
}

\author{
Ocular ischaemic syndrome associated with neovascular glaucoma: a case report
}

Enyr Saran Arcieri ${ }^{1}$

Vital Paulino Costa ${ }^{1,2}$
${ }^{1}$ Departamento de Oftalmologia, Setor de Glaucoma, Hospital das Clínicas da Universidade Estadual de Campinas - UNICAMP.

${ }^{2}$ Departamento de Oftalmologia, Hospital das Clínicas da Faculdade de Medicina da Universidade de São Paulo.

Os autores não tem interesse comercial nas drogas citadas no presente artigo.

Endereço para correspondência: Enyr Saran Arcieri. Rua Corcovado, 155 / 402 - Uberlândia (MG) CEP 38411-092. E-mail: esaran@terra.com.br

\section{RESUMO}

Os autores apresentam um caso de glaucoma neovascular secundário à síndrome ocular isquêmica como manifestação inicial de doença oclusiva de artérias carótidas. O exame de Doppler colorido das carótidas e órbitas mostrava presença de todos os fatores associados a um maior risco de desenvolver a síndrome: estenose carotídea grave bilateral, alto grau de estenose da artéria carótida interna e fluxo reverso na artéria oftálmica. Os autores discutem o fato do glaucoma neovascular ter sido controlado com apenas uma medicação tópica.

Descritores: Glaucoma neovascular/etiologia; Estenose carotídea/complicações; Isquemia; Corpo ciliar; Artérias carótidas; Artéria oftálmica; Relato de caso

\section{INTRODUÇ̃̃̃O}

A síndrome ocular isquêmica (SOI) resulta de uma hipoperfusão ocular prolongada causada por uma grande estenose ou oclusão da artéria carótida interna extracranial ou da artéria carótida comum. Geralmente acomete indivíduos do sexo masculino e com mais de 50 anos de idade ${ }^{(1-2)}$, associando-se freqüentemente a alterações sistêmicas severas e alta taxa de mortalidade, com envolvimento bilateral em $20 \%$ dos $\operatorname{casos}^{(2)}$.

A SOI é caracterizada por manifestações no segmento anterior, incluindo dilatação das veias episclerais, edema corneano, células e "flare" na câmara anterior, pupila em meia midríase e pouco reagente, catarata e neovascularização de íris e ângulo. As manifestações no segmento posterior incluem dilatação venosa, hemorragias intrarretinianas em média periferia e microaneurismas, neovascularização do disco e/ou retina, estreitamento arteriolar, tortuosidade vascular e edema macular ${ }^{(3)}$. Alguns pacientes relatam dor constante localizada na região orbitária, que irradia anteriormente, piora quando a cabeça é elevada e melhora quando é abaixada.

Há desenvolvimento de glaucoma neovascular em 35\% dos $\operatorname{casos}^{(2)}$, embora em alguns pacientes o valor da pressão intra-ocular (PIO) permaneça dentro dos limites da normalidade ou até mesmo reduzido, apesar do fechamento angular completo. O objetivo deste relato é apresentar um caso de SOI que foi submetido à investigação com Doppler colorido e apresentou glaucoma neovascular controlado com medicação tópica.

\section{RELATO DE CASO}

Paciente de sexo masculino, branco, 77 anos, queixava-se de dor e hiperemia conjuntival em OD há 1 dia, com piora da acuidade visual (AV) há 
1 mês. O paciente era hipertenso há 15 anos, estando em uso de hidroclorotiazida e nimodipina, porém não era diabético. Havia sido submetido à facectomia extracapsular com implante de lente intra-ocular em OD há 5 meses e havia apresentado $\mathrm{AV}=0,5$ neste olho com melhor correção 3 meses após cirurgia.

Ao exame oftalmológico, o paciente apresentava AV corrigida de $\mathrm{CD}$ (conta dedos) a $1 \mathrm{~m}$ em OD e 0,5 no OE. Os reflexos pupilares revelavam defeito pupilar aferente em OD. À biomicroscopia, o OD apresentava hiperemia conjuntival 2+/4+, córnea com edema microbolhoso difuso $1+/ 4+$, pseudofacia, LIO tópica e centrada com pigmentos na superfície, área de rotura de cápsula posterior superiormente, sinéquia anterior em região superior e temporal, pupila redonda com ectrópio de úvea, íris com neovasos difusos (Figura 1), câmara anterior formada, sem reação inflamatória. O OE apresentava catarata nuclear $2+/ 4+$.

À gonioscopia, observava-se ângulo fechado $360^{\circ} \mathrm{com}$ goniossinéquias no OD, e no OE, notou-se ângulo aberto $360^{\circ}$. À tonometria de aplanação apresentava PIO de $44 \mathrm{mmHg}$ em $\mathrm{OD} \mathrm{e} 19 \mathrm{mmHg}$ em OE. À oftalmoscopia indireta, observava-se no OD retina aplicada, mácula sem alterações, papila normal, escavação fisiológica $(0,3 \times 0,4)$, cruzamentos $\mathrm{AV}$ patológicos, áreas de hemorragia intrarretiniana em média periferia, estreitamento arteriolar, tortuosidade vascular, ausência de sinais de oclusão venosa ou arterial. O OE não apresentava alterações.

Inicialmente, prescreveu-se maleato de timolol 0,5\% 12/ $12 \mathrm{~h}+$ brimonidina $8 / 8 \mathrm{~h}+$ atropina $1 \% 8 / 8 \mathrm{~h}+$ acetazolamida $250 \mathrm{mg}$ VO 6/6h. Após 1 semana, apresentava $\mathrm{PIO}=16 \mathrm{mmHg}$ e $\mathrm{AV}=0,1 \mathrm{em} \mathrm{OD}$, o que permitiu a suspensão da acetazolamida e da brimonidina.

Realizou Doppler colorido de carótidas e órbitas que evidenciou: obstrução total da artéria carótida interna direita (Figura 2A), estenose estimada em 80 a 99\% em artéria carótida interna esquerda, ausência de fluxo nas artérias vertebrais e fluxo reverso na artéria oftálmica direita (Figura 2B).

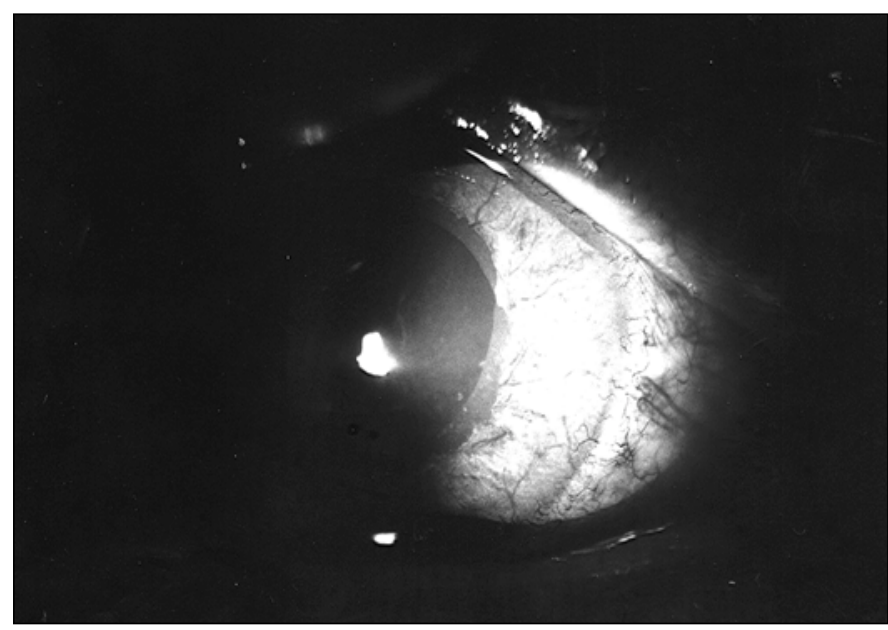

Figura 1 - Olho direito mostrando hiperemia conjuntival, neovascularização iriana e ectrópio uveal

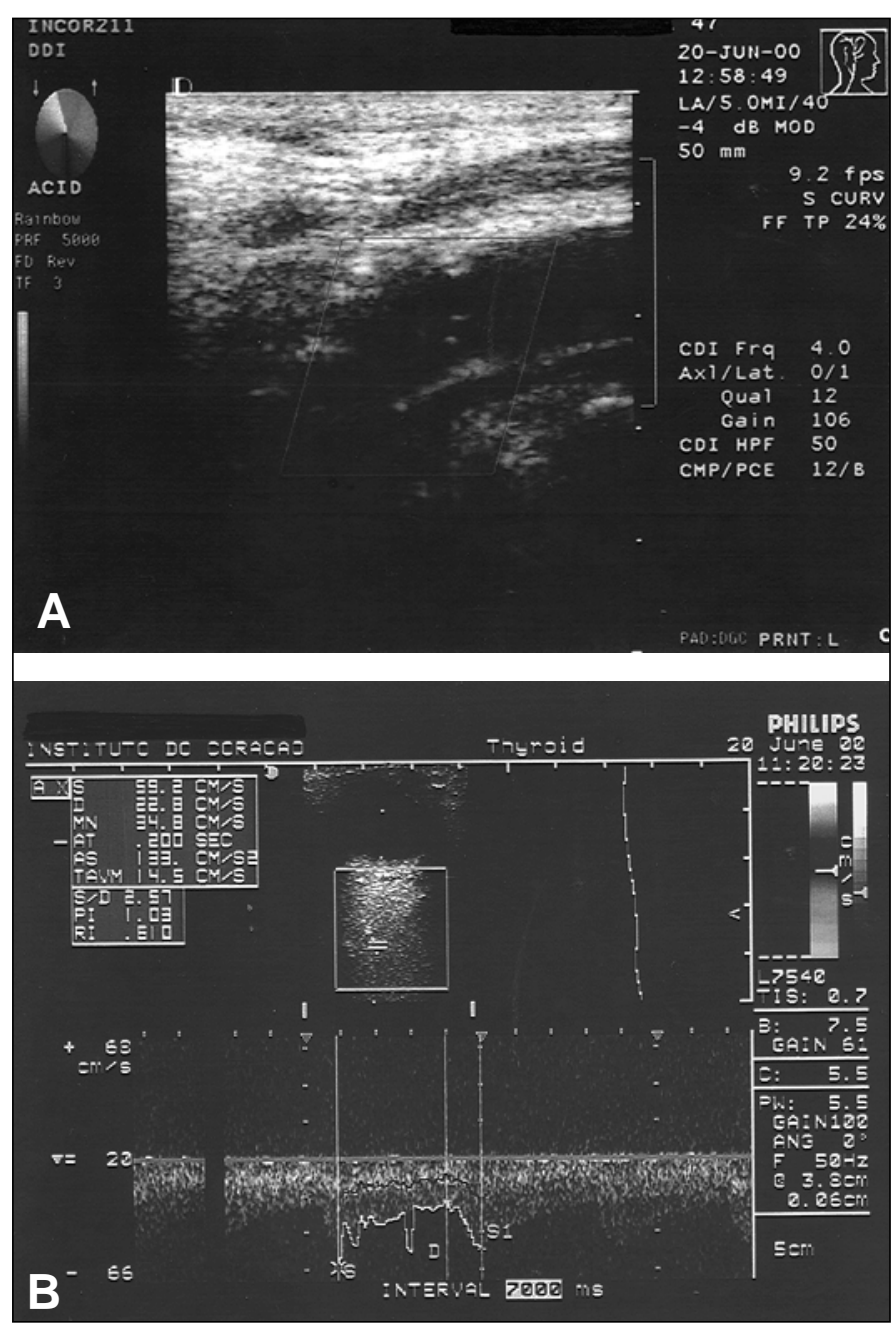

Figura 2-Doppler colorido. 2A. Mostrando oclusão total de artéria carótida interna direita. 2B. Mostrando fluxo reverso na artéria oftálmica direita

O paciente foi encaminhado para realizar panfotocoagulação retiniana em OD, tendo sido submetido a 3 sessões. Na última sessão, apresentou sangramento retiniano que evoluiu para hemorragia vítrea. Após três meses, houve reabsorção total da hemorragia. Atualmente, apresenta $\mathrm{AV}$ no $\mathrm{OD}=\mathrm{CD}$ a $4 \mathrm{~m}, \mathrm{PIO}=16 \mathrm{mmHg}$ em uso de maleato de timolol $0,5 \% 12 / 12 \mathrm{~h}$ e regressão dos neovasos de íris. O OE não apresenta nenhum sinal ou sintoma de isquemia ocular, estando com AV e PIO estáveis, sem uso de medicamentos.

\section{DISCUSSÃO}

A doença oclusiva da artéria carótida associada a SOI constitui-se na $3^{\text {a }}$ causa mais comum de glaucoma neovascu$\operatorname{lar}^{(4)}$. O prognóstico visual nestes casos é pobre. Um estudo mostrou que, mesmo quando vários pacientes apresentavam inicialmente uma visão relativamente preservada $(43 \% \mathrm{com}$ AV entre $20 / 20$ e $20 / 50 ; 20 \%$ entre $20 / 60$ e $20 / 400$ ), quase $60 \%$ progrediram para acuidade visual de conta dedos ou pior após 
1 ano, mesmo com terapêutica máxima ${ }^{(5)}$. A presença de neovascularização da íris é um indicador importante de mau prognóstico visual. No mesmo estudo, a visão de $82 \%$ dos pacientes que apresentavam "rubeosis" foi de conta dedos ou pior após 1 ano de seguimento ${ }^{(5)}$.

O exame de imagem com Doppler colorido permite uma análise qualitativa e quantitativa do fluxo sangüíneo retrobulbar. O primeiro estudo a descrever o uso do Doppler colorido para avaliar a circulação retrobulbar em pacientes com doença oclusiva da artéria carótida foi publicado por Lieb et $\mathrm{al}^{(6)}$, que observaram uma redução na velocidade do fluxo sangüíneo nas artérias oftálmica e ciliar posterior curta temporal de um paciente com oclusão da artéria carótida interna.

Em um estudo de Costa et al.(7), a média de estenose da artéria carótida em pacientes com SOI $(96,2 \pm 6,1 \%)$ foi significativamente maior que em pacientes com doença oclusiva severa da artéria carótida sem SOI $(87,2 \pm 8,2 \%)$. A ocorrência de estenose bilateral severa $(>70 \%)$ foi mais freqüente em pacientes com SOI, sugerindo que o fluxo na artéria carótida interna contralateral pode ser importante para compensar a redução do fluxo sangüíneo ispsilateral (via polígono de Willis). Outros autores já haviam sugerido que a SOI é observada em pacientes que apresentam mais de $90 \%$ de estenose da artéria carótida interna, especialmente quando a oclusão é bilateral ${ }^{(8)}$.

Estudos com Doppler ${ }^{(9)}$ têm indicado que em pacientes com doença oclusiva severa da artéria carótida interna ocorre um fluxo reverso na artéria oftálmica (via artéria carótida externa) quando o polígono de Willis é insuficiente para prover fluxo sangüíneo cerebral adequado. Uma alta incidência de fluxo reverso na artéria oftálmica tem sido encontrada em pacientes com SOI, variando entre 66,7 e $83,3 \%{ }^{(10)}$.

Costa et al. observaram que pacientes com estenose carotídea severa bilateral, fluxo reverso na artéria oftálmica e com alto grau de estenose da carótida interna apresentam maior risco de desenvolver $\mathrm{SOI}^{(7)}$. A associação entre fluxo reverso na artéria oftálmica e SOI é explicada graças a um fenômeno de "roubo", caracterizado por um "shunt" com o circuito intracraniano de baixa resistência e redução do fluxo sangüíneo retrobulbar $^{(11)}$. De fato, pacientes com fluxo reverso na artéria oftálmica apresentam velocidades de fluxo inferiores nas artérias central da retina e ciliares posteriores curtas quando comparados a pacientes com o mesmo grau de estenose carotídea e fluxo anterógrado na artéria oftálmica ${ }^{(11)}$.

O paciente em questão apresentou sinais característicos da SOI ao diagnóstico, com alterações nos segmentos anterior e posterior. Os diagnósticos diferenciais mais importantes neste caso incluem retinopatia diabética (onde as hemorragias se concentram no polo posterior, além da presença de outros achados, como exsudatos e neovasos retinianos), e oclusão de veia central da retina (onde as hemorragias se distribuem por toda a retina) $)^{(1)}$.

Através do exame de Doppler colorido das carótidas e órbitas, observamos que ele possuía todos os fatores associados a um maior risco de desenvolver SOI, tais como estenose carotídea severa bilateral, fluxo reverso na artéria oftálmica e alto grau de estenose da artéria carótida interna.
Outro fato relevante é o de que, apesar de apresentar seu seio camerular totalmente sinequiado, sua PIO permaneceu controlada apenas com o uso de um medicamento tópico (beta bloqueador). Isto é justificado pela hipoperfusão do corpo ciliar secundária à oclusão total da artéria carótida interna direita com subseqüente redução da produção do humor aquoso e provável hipersensibilidade ao uso de maleato de timolol. Nestes casos, o restabelecimento cirúrgico do fluxo sangüíneo carotídeo e orbitário após endarterectomia resulta em importante elevação da PIO no pós-operatório, presumivelmente pela melhora da perfusão do corpo ciliar e aumento da produção de humor aquoso ${ }^{(12)}$.

\section{ABSTRACT}

The authors present a case of neovascular glaucoma secondary to ocular ischemic syndrome as the initial manifestation of carotid occlusive disease. Color Doppler imaging of the carotids and orbit showed all the factors associated with a greater risk of developing this syndrome: severe bilateral carotid stenosis, high-grade carotid stenosis, and reversed ophthalmic artery blood flow. The authors also discuss how the neovascular glaucoma was controlled with only one topical medication.

Keywords: Neovascular glaucoma/etiology; Carotid stenosis/ complications; Ischemia/ocular; Ciliary body; Carotid arteries; Ophthalmic artery; Case report

\section{REFERÊNCIAS}

1. Garrido Neto TL, Halfeld R, Takahashi WY. Síndrome ocular isquêmica: relato de caso. Arq Bras Oftalmol 1998;61:238-41.

2. Brown GC, Magargal LE. The ocular ischemic syndrome. Clinical, fluorescein angiographic and carotid angiographic features. Int Ophthalmol 1988;11: 239-51.

3. Dugan Jr. JD, Green WR. Ophthalmologic manifestations of carotid occlusive disease. Eye 1991;5(Pt 2):226-38.

4. Shields MB. Glaucomas associated with disorders of the iris and ciliary body. In: Shieldes MB. Textbood of glaucoma. 4th ed. Baltimore: Williams \& Wilkins; 1997. p. 14.

5. Sivalingam A, Brown GC, Magargal LE. The ocular ischemic syndrome. III. Visual prognosis and the effect of treatment. Int Ophthalmol 1991;15:15-20.

6. Lieb WE, Flaharty PM, Sergott RC, Medlock RD, Brown GC, Bosley T, Savino PJ. Color Doppler imaging provides accurate assessment of orbital blood flow in occlusive carotid artery disease. Ophthalmology 1991;98:548-52.

7. Costa VP, Kuzniec S, Molnar LJ, Cerri GG, Puech-Leao P, Carvalho CA. Clinical findings and hemodynamic changes associated with severe occlusive carotid artery disease. Ophthalmology 1997;104:1994-2002.

8. Tatemichi TK, Chamorro A, Petty GW et al. Hemodynamic role of ophthalmic artery collateral in internal carotid artery occlusion. Neurology 1990;40(3 Pt 1):461-4.

9. Brown GC, Magargal LE, Simeone FA, Goldberg RE, Federman JL, Benson WE. Arterial obstruction and ocular neovascularization. Ophthalmology 1982;89(2):139-46.

10. Costa VP, Carvalho CA, Kuzniec S, Molnar LJ, Puech-Leão P. Doença oclusiva das artérias carótidas: alterações oculares e repercussão hemodinâmica sobre a circulação retrobulbar. Arq Bras Oftalmol 1997:60:399. [Resumo 83]

11. Costa VP, Kuzniec S, Molnar LJ, Cerri GG, Puech-Leao P, Carvalho CA. Collateral blood supply through the ophthalmic artery: a steal phenomenon analyzed by color Doppler imaging. Ophthalmology 1998;105:689-93.

12. Edwards MS, Chater NL, Stanley JA. Reversal of chronic ocular ischemia by extracranial-intracranial arterial bypass: case report. Neurosurgery 1980;7:480-3. 